\title{
A Stimulated Simulation System Based on Ovation Virtual DCS Shihe $\mathrm{CHEN}^{1, a}$, Fengping $\mathrm{PAN}^{2}$, Lingling $\mathrm{SH}^{3}$, Zhiqiang $\mathrm{PANG}^{4}$, Juanjuan $\mathrm{REN}^{5}$, Xiangsen $\mathrm{ZHAN}^{6, \mathrm{~b}}$ \\ 1,2,3,4,5Electric Power Research Institute of Guangdong Grid Company, Guangzhou 510080, China \\ ${ }^{6}$ UNI Group (Beijing) Intelligent Control Science \& Technology Co., Ltd., Beijing 100085, China \\ bemail: zhanxiangsen@unicst.com
}

Keywords: Stimulated simulation; Virtual DCS; OPC; Ovation

\begin{abstract}
In full accord function with reality power unit, Operator station and engineer station provided by Ovation virtual DCS is accepted by simulation users. The development of interface between virtual DCS and simulation model is key technology for Stimulated Simulation System. The application in Guangdong Gao Lan Gang power plant of a stimulated simulation System Based on Ovation Virtual DCS is introduced here. Key technology of Communication Interface is discussed in this paper.
\end{abstract}

\section{Introduction}

To improve the operators' technical skill level in the power plant, a large number of simulation training and practice in the real environment is a necessary[1]. However, training directly in the field environment is not only unsafe, but also unable to practice all of the running programs. So in a long time, high-end training is the hard stimulated simulation training which is based on purchasing a set of real DCS control system. But it has not been able to widely promote because of the high cost.

As leading manufacturers of control system gradually launched their own virtual DCS system, since the virtual DCS simulation training system interface is authentic and friendly and the cost has greatly reduced relative to using real hardware controller of hard stimulation, so in recent years, the market share of soft incentive simulation system based on virtual DCS increases year by year.

The application of the Ovation3.3.1 Virtual DCS in Zhonghai Gao Lan Gang power plant is an example in this paper, specifying the system structure and interface communication mode of the stimulated simulation System based on Ovation virtual DCS, as well as some key points in the process of project implementation[2].

\section{Simulated simulation training system}

A. The Classification of Simulated Simulation Training System

Simulated simulation training system using the same function interface as the operator station for training students, its operating interface and operation mode are the same as the real power plant, and it is widely accepted by simulation user. According to different control logic implementation mode, the simulative simulation system can be divided into hard stimulation, half stimulation and soft stimulation.

Hard stimulation: Hardware configuration of the whole control system are the same as the power plant, operator station uses real operator station software, and all control logic is computed by using real DPU. The data between simulation model and control system is exchanged through the actual I/O communication card, or through the special communication interface card.

Half stimulation: The real operator station software is used only in operator station, simulation model and control logic are implemented by manufacturer. Interface software, in fact, only exchanges operating instructions and status data with operator station.

Soft stimulation: The real operator station software is used by operator station, and in this mode 
control logic is computed by virtual DPU (i.e., the simulation controller software), which is different with the hard stimulation, but its operating process and mode are identical with real power plant for terminal or thermal operator.

B. The Advantages and Disadvantages of Simulated Simulation System

In manufacturing enterprises, the disadvantage of simulative simulation system is not obvious, but it is obvious for training center or technical colleges and universities, because there are numerous students in the training center or technical colleges, and group training is provided by using the same model, while simulated simulation system does not have this kind of condition unless extra control system virtual software packages is purchased.

C. The Control System Virtual Software Package of Soft Stimulation

For soft stimulation, the manufacturer need to provide complete control system virtual software package. Depending on the differences of manufacturer, its composition is different, which may include, but not limited to, the simulation server software, the simulation controller software, the operator station and engineer station software, history station software and database software, etc.

The current domestic and international major manufacturers have provided virtual software packages(Emerson, ABB, Hollysys, Mitsubishi, Xinhua XDC800, GE, etc.), which are used for control system simulation in the field of industrial control, and docking with simulation model developed by suppliers, to provide users with real friendly interface of training.

\section{Soft incentives simulation system architecture based on Ovation virtual package}

Ovation virtual software can realize some functions of Ovation control system such as the logic operation, screen monitoring, alarm monitoring, historical data storage and central database management, etc., as shown in figure 1.

Soft simulated simulation system is composed of three parts, control system virtual package, device model simulation software and interface station:

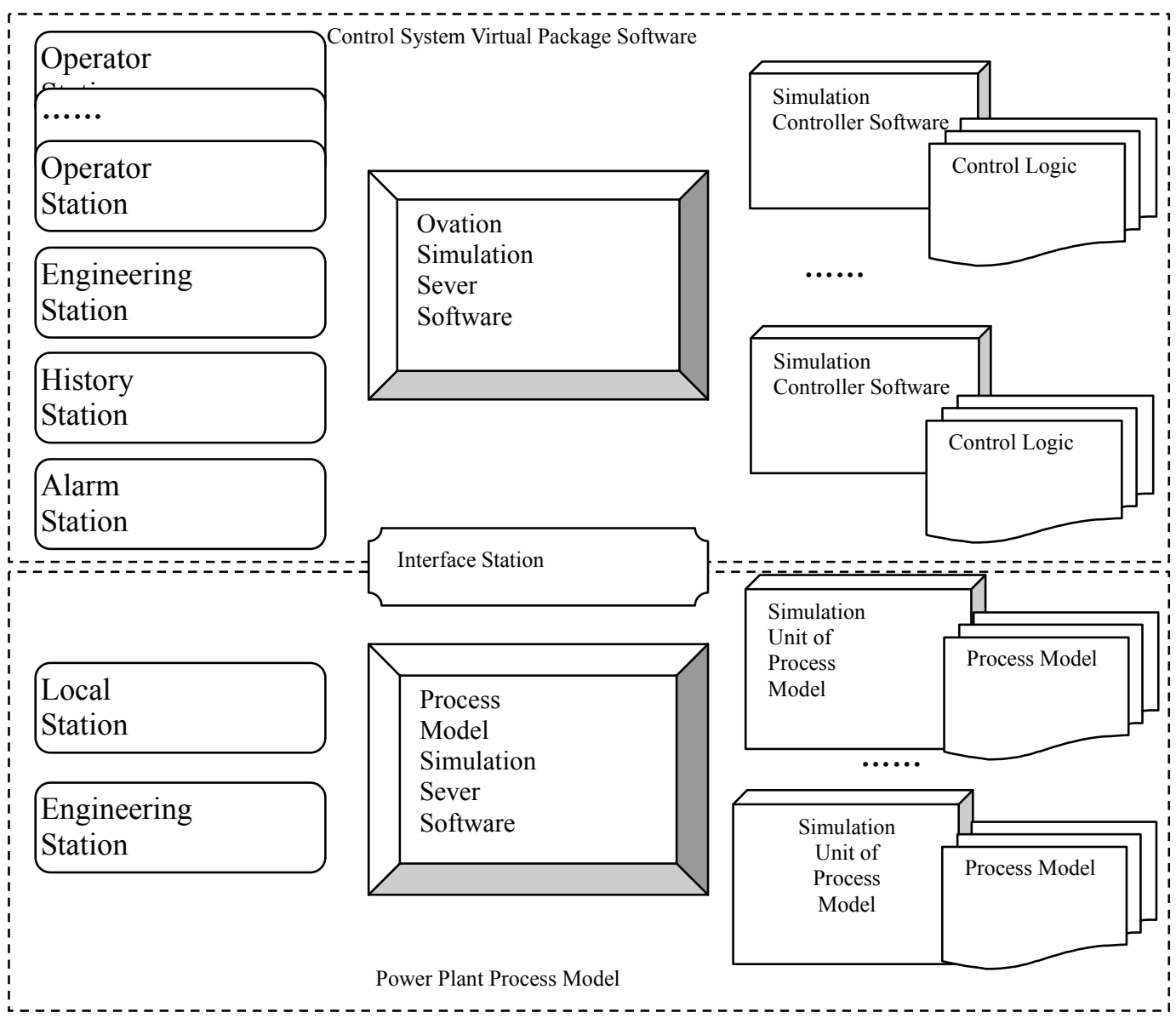

Fig.1. System structure

In control system virtual package, the database can load the data points library of the power plant 
directly, the simulation controller can load control logic on site, the operator station can use the site monitoring screen, so as to simulate all the calculation, monitoring and operation of the site control system.

In device model simulation software, the calculating element of model performs each device simulation model of subsystem, and summarizes the data to simulation server.

The communication function of interface station includes two parts:

Data communication function: It is equivalent to the hardware $\mathrm{I} / \mathrm{O}$ channel (including sensors and actuators) and intelligent equipment data communication pipeline of the worksite. Send the status data of simulation model to the control system, and then send the control instruction to equipment simulation model after the Ovation control system receives that.

Signaling channel: Send the control instruction to Ovation simulation server, these instructions including the start and operation of the controller, the storage and calling of the working condition, the acceleration or deceleration of the calculating rate, etc.

\section{The interface technology of soft incentive simulation system based on Ovation virtual package}

Ovation virtual package provides two modes to communicate with external systems, namely API mode and OPC mode[3].

\section{OPC Server}

Ovation providing OPC server (usually deployed on the communication interface station drop180), users can access the OPC server on the station through any OPC client program, thus can read and write the data of Ovation control system by using the OPC standard protocol.

Ovation Simulation API

Namely SIMAPI. The API provides the interface between simulation model and Ovation system, and the interface can be used to read or write the real time value of data points in Ovation system. SIMAPI contains three sublibraries: SIM REGISTER, SIM COMMAND and SIM I/O.

1) SIM COMMAND library: The family of library functions, which send control commands to the Ovation virtual package, includes running, freezing, saving and loading conditions, changing the running speed, etc.

2) SIM REGISTER library: The third party can use the library function to register on the Ovation simulation server, and the third party applications can receive notifications of various kinds of SIM COMMAND when the Ovation simulation server system changes.

3) SIM IO library: The library is a family of functions, which can use SIM IO for data communication in SIMAPI. Firstly need to define the table of simulated point configuration information, then read and write the data or data state by using various functions.

OPC server can be used only for data communication, so there are two methods for complete communication plan.

Only API mode: Only use SIMAPI for interact instructions and data communications.

Blend mode: The OPC protocol is used for data communications with Ovation simulation server, and SIMAPI is used to send interactive instruction to Ovation server[4]. 


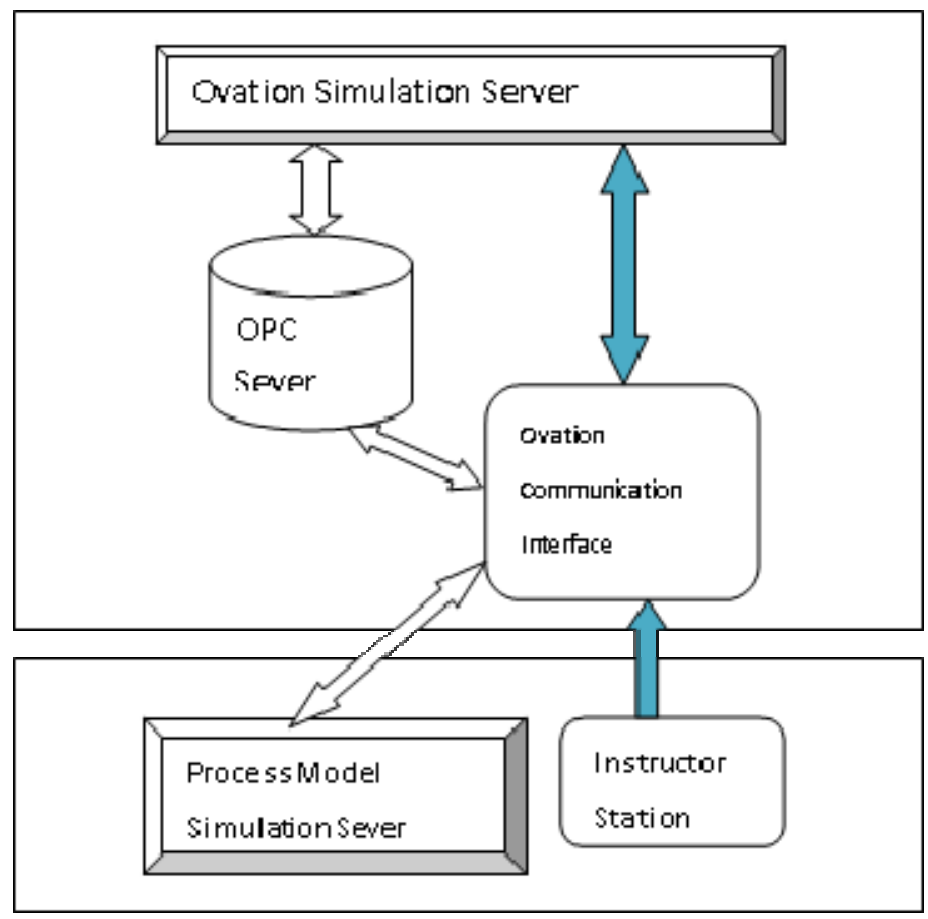

Fig.2. System structure

This project uses the blend mode (as shown in Figure 2), its advantage lies in not need to organize the table of point configuration information, according to the requirement of the SIM IO library:

The interface program receives the instructions of instructor station from equipment simulation model, and transmits the instructions to Ovation simulation server, to control the operation of the Ovation simulation server to control logic.

The interface program will send the model status information data of simulation server to the OPC server deployed on interface station, and also send the instruction data in OPC sever to model simulation server. The data synchronization between OPC server and Ovation simulation sever is processed by Ovation package.

\section{The implementing points of soft incentive simulation system based on Ovation virtual package}

\section{A. The Name of Data Communication Point s}

For the control command communication points, such as AO and DO, interface program directly reads the corresponding data from the OPC server, and sends to model simulation the server.

For the equipment state communication points, such as AI and DI, can not be processed like above. Usually build a new point on interface station (general Drop180), which call for actual logic point (such as BMS01A001) and add a prefix (if the agreed prefix is FZ, the new point on interface station is FZBMS01A001). And in the Config tab of actual logic point information panel, the new point should been configured[5].

B. The Logic Compensation Algorithm

For the data of steam flow, feed flow rate and air volume, most of them is compensated and corrected in logic, and the correct value is unable to get if the data sent to control system is calculated directly by model. There are several plans for this problem:

Change logic, skip the logic of compensation algorithm.

Backstep the formula of compensation algorithm, and then collate the point.

If the point is complex and the formula cannot be backstepped, then the piecewise function may be used for fitting.

Modify the logic of the program is the least desirable. When the version of the logic is updated in the real power plant, and it need to be resumed on the simulator at the same time, the logic also need to be modified manually on the simulation system. In this project backstepping formula is adopted. 


\section{The Field Bus Device Point}

Field bus has been gradually applied in the power plant. The points sent to DCS through the bus are usually packaged type (LP), which requires the points of simulation model and control system communication are packaged points as well, otherwise the control logic need to be modified. In this project the process that the state information of multiple equipments is encapsulated as a packaged points is implemented in the device model, thus only OPC is used for the communication of packaged point.

D. Questions about Interactive Instruction

In theory, after receiving the instruction of instructor station, the communication interface can forwarding the instruction to the Ovation simulation server directly, but we found that, in the process of debugging, Ovation simulation virtual package does not store the interface data points while the operation condition is stored. Therefore, the state from the Ovation operator station does not match that of condition storage when communication interface send the instruction of load condition to Ovation simulation server, in order to solve this problem, we modify that the communication interface will send the state data to OPC server after sending the instruction of loading condition[6].

\section{Conclusion}

A stimulated simulation System is gradually favored by the customers for its authenticity and friendliness. However, the disadvantage is also obvious, it will occupy more resources and the group training can't be provided under a set of resource, what's more, it will cost too much. Therefore, it is more suitable for owner units, such as power plants, chemical plants, etc., or scientific research institutes that research the control strategies, while it is not very appropriate for training center or technical colleges.

\section{References}

[1] Lv Chongde, Ren Tingjin, Jiang Xuezhi,etc. "Simulation and modeling of large thermal power unit system"[M]. Tsinghua university press, 2002

[2] "MUSE user manual, UNI group(Beijing) intelligenct control science \& technology co.", LTD., 2013

[3] "Simulation User Guide for Ovation 3.3.1. Version 1", July 2010, Emerson

[4] "Virtual DCS technology and real-time system interconnecting application in large scale power plant", VOL.35 NO1, Jan.2005. Journal of Southeast University

[5] "Method of External Expansion Function Based on OVATION Control System", VOL.26 NO10, 2013, Industrial Control Computer

[6] "MUSE user manual, UNI group(Beijing) intelligenct control science \& technology co.", LTD., 2014 